Modeling planetary interiors in laser based experiment using shockless compression

J. Hawreliak, J. Colvin, J. Eggert, D. Kalantar, H. E. Lorenzana, S. Pollaine, J. Stolken, B. A. Remington, K. Rosolankova, J. S. Wark

May 7, 2006

HEDLA 2006'

Houston, TX, United States

March 11, 2006 through March 14, 2006 
This document was prepared as an account of work sponsored by an agency of the United States Government. Neither the United States Government nor the University of California nor any of their employees, makes any warranty, express or implied, or assumes any legal liability or responsibility for the accuracy, completeness, or usefulness of any information, apparatus, product, or process disclosed, or represents that its use would not infringe privately owned rights. Reference herein to any specific commercial product, process, or service by trade name, trademark, manufacturer, or otherwise, does not necessarily constitute or imply its endorsement, recommendation, or favoring by the United States Government or the University of California. The views and opinions of authors expressed herein do not necessarily state or reflect those of the United States Government or the University of California, and shall not be used for advertising or product endorsement purposes. 


\title{
Modeling planetary interiors in laser based experiments using shockless compression.
}

\author{
J. Hawreliak \\ J. Colvin J. Eggert \\ D. H. Kalantar \\ H.E. Lorenzana \\ S. Pollaine \\ K. Rosolankova \\ B.A. Remington \\ J. Stölken \\ J.S. Wark
}

November 10, 2006

\begin{abstract}
X-ray diffraction is a widely used technique for measuring the crystal structure of a compressed material. Recently, short pulse x-ray sources have been used to measure the crystal structure in-situ while a sample is being dynamically loaded. To reach the ultra high pressures that are unattainable in static experiments at temperatures lower than using shock techniques, shockless quasi-isentropic compression is required. Shockless compression has been demonstrated as a successful means of accessing high pressures. The National Ignition Facility (NIF), which will begin doing high pressure material science in 2010, it should be possible to reach over $2 \mathrm{TPa}$ quasi-isentropically. This paper outlines how x-ray diffraction could be used to study the crystal structure in laser driven, shocklessly compressed targets the same way it has been used in shock compressed samples. A simulation of a shockless laser driven iron is used to generate simulated diffraction signals. And recently experimental results are presented.
\end{abstract}

\section{Introduction}

Models of planetary interiors rely on accurate laboratory measurements of equations of state [1]. High pressure measurements are generally limited by the experimental apparatus. Diamond anvil experiments can be used to study statically compressed samples up to only a few hundred GPa [2]. Dynamic techniques are needed to reach pressures exceeding $4 \mathrm{TPa}$ that exist at the cores of the gas giants [3]. These pressure should be obtainable in the next generation of high energy density experimental facilities such as the National Ignition Facility (NIF) [4].

This paper discusses how the crystal structure can be measured in dynamically compressed samples using in-situ x-ray diffraction $[5,6,7]$. First, a brief discussion of shock and shockless compression is presented as a means of obtaining high pressures. Then we describe how the x-ray diffraction techniques would differ between the shock and shockless drive. Finally a comparison of data from a recent experiment are compared with the initial shock compression of the sample.

\section{Dynamic compression techniques}

Shock compression has been used over the last half century to provide a wealth of information about materials at high pressures [11]. Traditionally experiments have been done using a gas gun to launch a projectile at a stationary target, or using high explosives (HE) to apply a sharp pressure pulse[12]. 
More recently, shock wave research has extended to large laser and z-pinch facilities. Even though the technology to create a shock is different in each method, the sample will always transform to a single temperature and pressure described by the shock Hugoniot, the curve in phase space that represents the shock response of a material. To study samples under the ultra high pressure conditions relevant to planetary interiors in the laboratory, dynamic compression techniques which do not produce a shock are required. These techniques are labeled as "quasi-isentropic" because they produce high pressures off the shock Hugoniot in materials while maintaining low temperatures using a sequence of small shocks.

Quasi-isentropic has been performed using pillow impactors on gas guns[12], temporal pulse shaping on z-pinch facilities[13], HE expanding across a vacuum gap [14] and a two component target on laser facilities $[15,16]$. In a similar fashion to the $\mathrm{HE}$ case, the laser target uses a plastic reservoir which is shocked with a high-powered laser and the plasma blow off from the ensuing blast wave crosses a vacuum gap and pressurizes the sample. The parameters of the target can be tailored to suit a particular compression regime. Unlike shock compression, the sample does not end up on a single point on the shock Hugoniot, but samples a continuum of states along the compression 'quasi-isentrope'. Where measurements made at different times look at different pressure and temperatures.

An example of a laser driven shockless compression target is shown in Fig. 1, and simulated pressure, temperature, and density profiles are shown in Fig. 2 for an on target drive laser intensity of $1 \times 10^{13} \mathrm{~W} / \mathrm{cm}^{2}$. This shows that over 5 to $10 \mathrm{~ns}$ period the pressure increases in the sample until it reaches a peak pressure, which is maintained for approximately $10 \mathrm{~ns}$. The beryllium layer serves as a heat shield to prevent the sample from melting on initial contact with the plasma, but ultimately a thermal wave causes the sample to heat up, setting up a large temperature gradient in the sample. The long time-scales and spatial gradients in the isentropically compressed case change the way the sample can be diagnosed using in-situ x-ray diffraction.

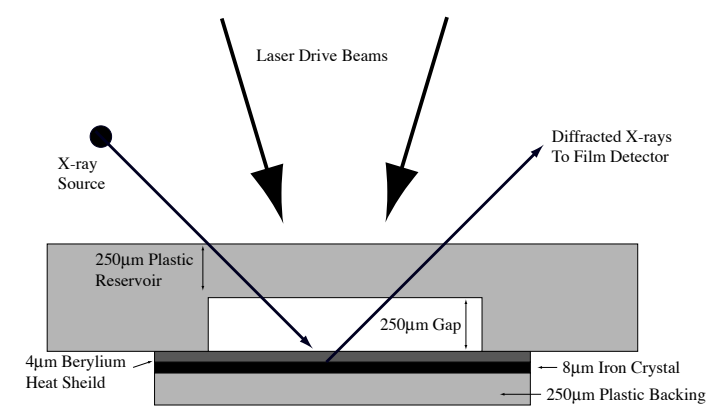

Figure 1: A schematic diagram of a shockless compression target. The drive beams are incident on the plastic reservoir, which causes the blow off from the back of the plastic to cross the gap and pile up on the iron sample. A thin layer of beryllium is used to prevent thermal contact between the iron sample and the plasma. 


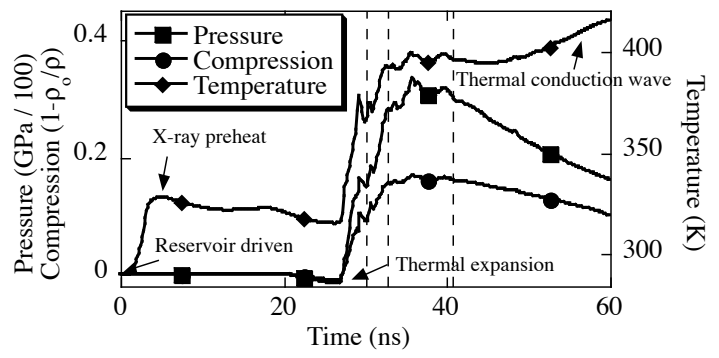

Figure 2: A plot of the simulated temperature, pressure and compression at the center of the $8 \mu \mathrm{m}$ iron foil from a 1D LASNEX simulation. The simulation shows slight preheating of the sample from the x-rays generated at the drive surface of the reservoir, causing a slight expansion before the pressure wave arrives at the center of the sample. The three lines indicate the time at which the simulated diffraction images are shown in Fig. 5

\section{X-ray diffraction from dynamically compressed materials}

With the development of x-ray diffraction of dynamic systems, $[6,7,17,18]$ it is possible to make a determination of crystal structure in-situ during the compression process. These in-situ measurements are critical to understanding how materials react under presssure as the crystallographic structure is fundamental in determining material properties. A change in crystal structure, known as a phase change, is usually accompanied by a dramatic change in bulk properties, i.e. volume collapse, change in electrical conductivity, change in magnetism, etc. We will discuss modifications to the existing wide angle x-ray diffraction techniques used on shock compression experiments [5] to make crystallographic measurements on quasi-isentropically compressed samples.

Figure 3 shows how the x-ray probes will differ between the two techniques. The gray shaded region denotes the time and the finite penetration depth of the x-ray probes on the crystal. In the shock case, shown in Figure 3 a), there is a discontinuity at the shock front between the uncompressed and shock compressed material. For x-ray diffraction this discontinuity means we can record a diffraction signal from both the unshocked sample and the shock compressed sample with a single pulse of x-rays which is time synchronous with the shock front reaching the material. The measurement of the unshocked signal is critical to making quantitative measurements because it provides a method for calibrating the experimental geometry of the detector and the initial crystal state.

In the isentropically compressed case, the compression happens over a longer timescale, as illustrated in Fig. 3 b). This means that it will require two separate x-ray pulses. One x-ray pulse is required to record a zero pressure signal. A second x-ray pulse is required to interrogate the sample at pressure. Unlike the shock case, where the shock front provides a discontinuity between compressed material and the zero pressure material, the isentropically compressed targets will have a continuous compression with large gradients into the sample. 

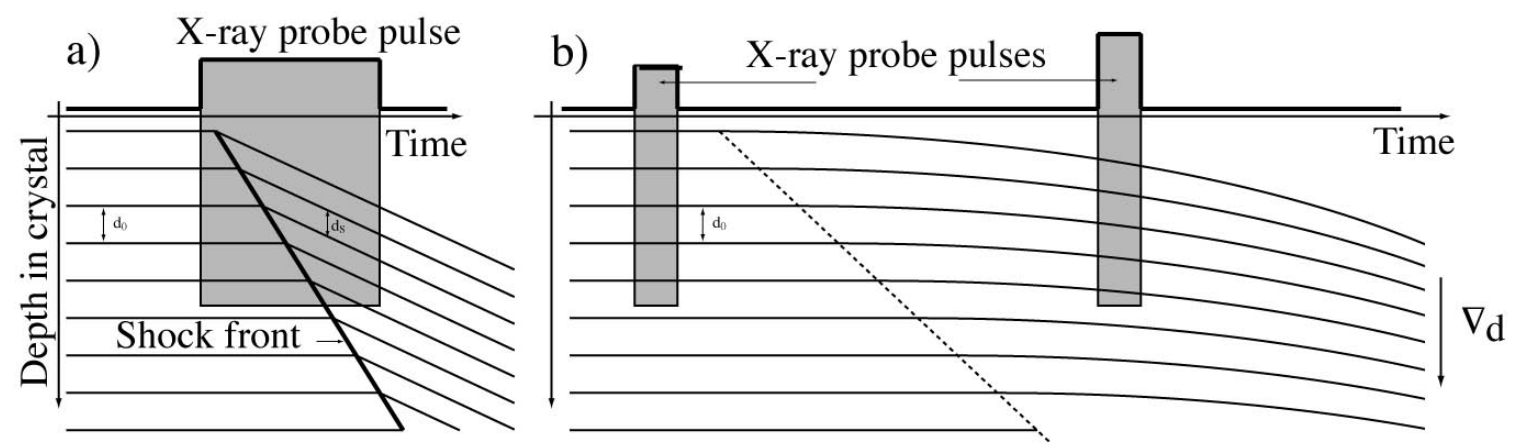

Figure 3: Schematic diagram showing a time-distance plot of representative lattice planes in iron that is compressed by a) shock loading and b) isentropic compression. The shaded region represents the x-ray probe time and depth in the crystal. Both the uncompressed and compressed lattice are probed by a single x-ray pulse in the shocked case, where as two separate pulses are required to probe in the isentropically compressed case. $d_{0}$ is the initial plane spacing, $d_{s}$ is the plane spacing in the shocked sample and $\nabla d$ is the direction of increasing plane spacing in the ramp compressed sample.

\section{Diffraction from Iron [100] crystals}

The properties of iron under high pressure and temperature have always been of interest owing to iron being a major component of the earth's inner core and its technological relevance to modern society. The $\alpha-\epsilon$ phase transition at $13 \mathrm{GPa}$, shown in Figure ?? is of particular importance to the shock physics community as it was first proposed by Bancroft et al. [19] to explain the discrepancy between Walsh's measurements [20] and Bridgman's original work up to $10 \mathrm{GPa}$ [21]. The crystallographic nature of the $\epsilon$ phase was not determined until later with static compression measurements using x-ray diffraction by Jamison and Lawson [22]. It was not until recent in-situ $\mathrm{x}$-ray diffraction experiments of laser shocked samples that this was confirmed[23]. With the recent success of in-situ x-ray diffraction in determining the crystal structure of shocked iron, we will use iron as the example to discuss the x-ray diffraction technique for isentropically compressed samples. We employ a simulation to show how the gradients in the sample will affect the width of the diffraction lines that will be recorded on the film.

Figure 4 shows how the compression of the iron varies in the sample at different times in the simulation. During the pressure ramp, $30 \mathrm{~ns}$ after the drive beams are incident on the reservoir surface, the density gradient is large going from ambient density to a compression of nearly $15 \%$. Since the x-rays can penetrate the entire sample, the diffracted peak will contain information from the full range of compressions at this time, and it will be significantly broadened. Close to the peak of the pressure pulse, $33.5 \mathrm{~ns}$, maximum compression is reached at the front surface, but there is still a large gradient in compression which will broaden the diffracted signal and make it difficult to extract detailed information.

Figure 5 illustrates line broadening effects due to the strain gradient in the iron sample. Figure 5 a) shows a line out from an experimental image from iron sample shock compressed with a laser using an iron K-shell backlighter ??. As stated before in the shock case we can probe several states of the material with a single pulse due to the penetration depth of the x-rays. In this case we 
get signal from pristine material (deepest in the sample), a compressed BCC lattice (intermediate phase) and the transformed HCP phase (closest to the surface). For comparison, Figure $5 \mathrm{~b}$ ) and c) show calculated signals at 3 times for iron and copper backlighters respectively. Two different examples are show for each time. The dashed lines in Fig. 5 b) and c) represent the diffraction peak profile dependent only on the variation in compression as a function of depth. The solid line includes some instrument broadening of the diffraction peaks. Figure $5 \mathrm{~b}$ ) shows that the diffraction signals recorded at 30 and 33.5 ns will be broaden significantly by the gradient in compression of the sample. The diffraction signal at $41.5 \mathrm{~ns}$ forms a tighter peak than the diffraction peak at the other two times, which can be attributed to the smaller gradient in the compression.

The broadening in the diffraction signal due to the strain gradient can be reduced by the choice of backlighter. When using $\mathrm{K}$-shell laser generated x-rays from an iron backlighter at $6 \mathrm{keV}$ the $1 / \mathrm{e}$ depth is about $7 \mu \mathrm{m}$ for the (002) plane, generating diffraction signal from nearly the entire sample. Alternatively the K-shell radiation from a laser based copper backlighter at $8 \mathrm{keV}$ the depth is reduced to less then $2 \mu \mathrm{m}$. Simulations of the diffraction peaks using a copper backlighter are shown in Fig. 5c). The copper backlighter, with its shallower penetration depth, generates sharper peaks than an iron backlighter but the scattering signal levels will be reduced due to the higher absorption in the iron at the copper k-shell emission line.

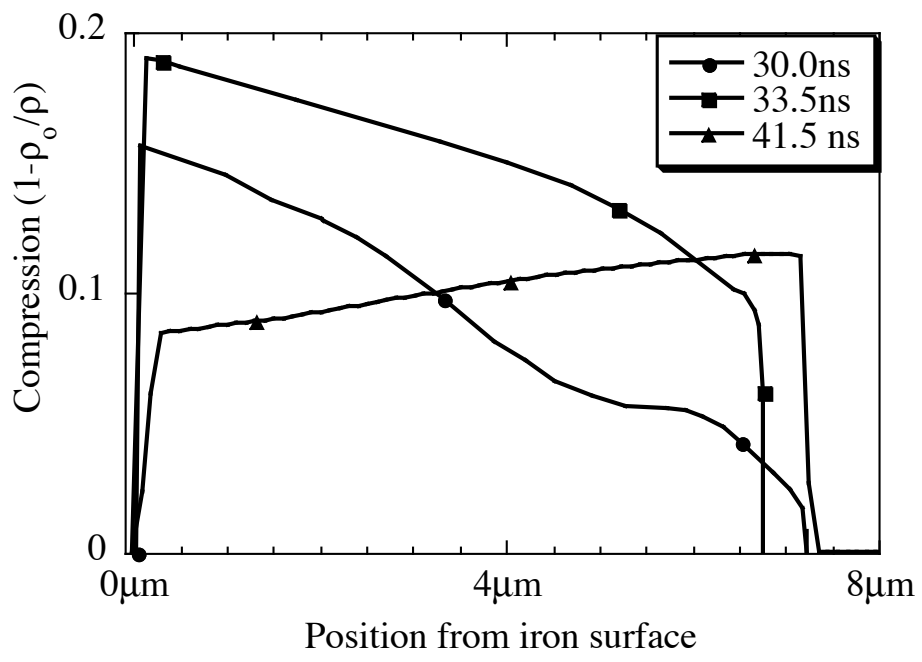

Figure 4: This plot shows the compression at different times through the sample. Both 30 and 33.5 ns show steep gradients in the compression. At 41.5 ns the compression is more uniform across the sample.

Figure 6 shows recent experimental data. The timing of the late x-ray pulse was set to probe the lattice as the compression wave reached the sample. In Figure 6 the data is obtained by a single late x-ray pulse. The two distinct peaks is consistent with the initial small shock that is generated by the ramped compression profile steepening up in the plastic heat shield layer. While a thick 


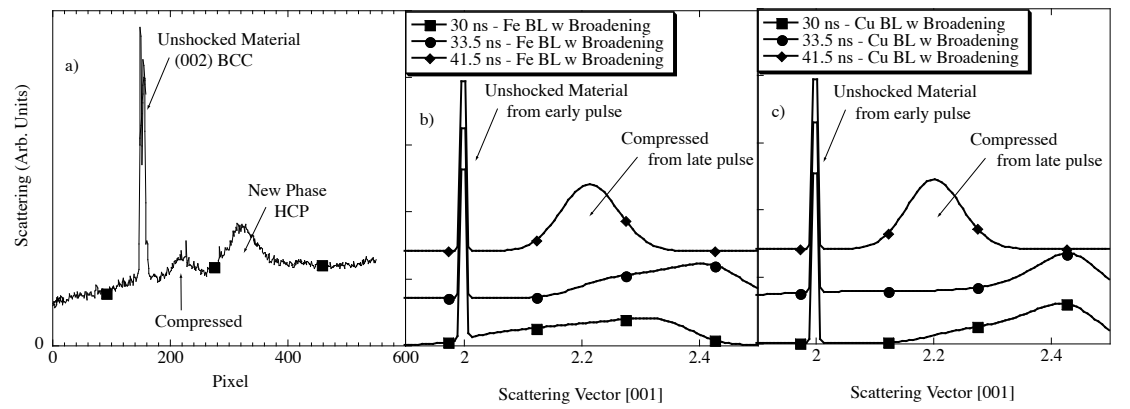

Figure 5: A comparison of line outs from a) raw experimental x-ray diffraction data from a shocked, single crystal sample and simulated diffraction using b) an iron backlighter and c) a copper backlighter. The shape of the simulated peaks in b) and c) reflect the range of compressions in the sample. The dashed curves use only the compression and depth to determine the peak shape, whereas the solid curves includes the broadening observed due to the $\alpha-\epsilon$ phase transition.
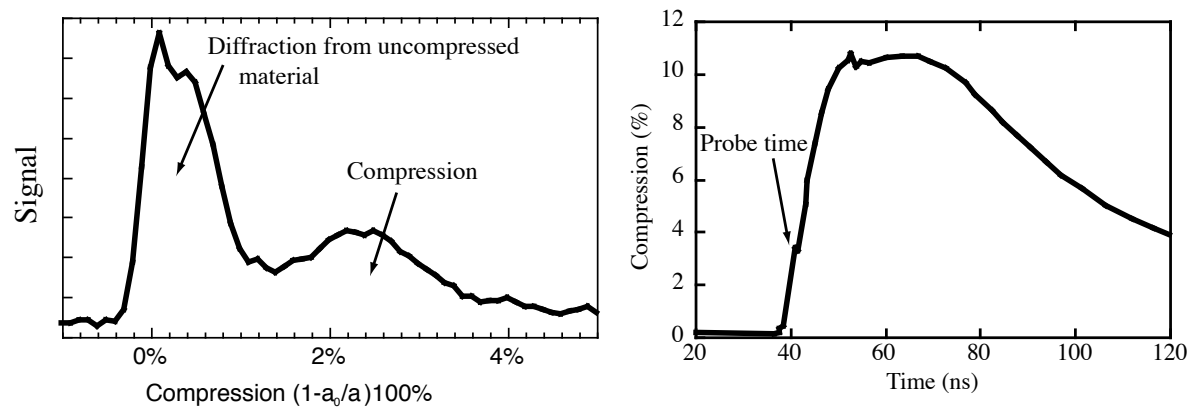

Figure 6: Experimental data and simulated temporal profile of the compression in the sample. The sample was Vanadium coated with a $5 \mu \mathrm{m}$ plastic heaat shield. The experimental data was generated using only the late time pulse, so the distinct peaks is consistent with a shocking of the sample.. 
heat shield over the sample is desired to keep the plasma heat from the sample, too thick a sample will lead to an initial shock or potentially shock compression of the sample.

\section{Conclusion}

Quasi-isentropic compression gives us the ability to explore material states off the shock Hugoniot. This approach also provides us with one key and truly unique capability, namely access to compressions in solids that approach the terapascal regime. Typically, single shock techniques melt solids at pressures exceeding 200-400 gigapascals, and so properties of solids beyond these pressures remain completely unexplored. Investigations in the terapascal regime enabled by quasi-isentropic compression certainly will provide an unprecedented window into the extreme states found in planetary interiors.

Time-resolved X-ray diffraction methodologies will play an unique and pivotal role in investigating the crystal lattice and other fundamental properties of the solid during these extreme conditions. For shocked solids, our group and others throughout the community have already demonstrated that x-ray diffraction can successfully probe atomistic phenomena such as phase transformations with nanosecond temporal resolution. However, diffraction measurements in a quasi-isentropically compressed solid have never been demonstrated and pose important challenges; in this work, we have discussed a novel approach to enabling such investigations. We have described key experimental aspects of a technique to perform time resolved diffraction measurements, including a new geometrical target design as well as relevant backlighter and timing issues. Additionally, we have explored the need for a detailed hydrodynamic understanding of the compression process, either through bulk measurements or detailed simulations, that will permit quantitative analyses of the quasi-isentropically compressed lattice. Finally, we have presented preliminary experimental results showing in situ, real-time lattice measurements during quasi-isentropic compression and model and quantify the contributions from spatial and temporal gradients. Development of this technique will continue on the Omega laser at the University of Rochester, the Jupiter Laser Facility at LLNL, and the Vulcan laser at the Rutherford Appleton Laboratory in the UK.

This work was conducted under the auspices of the U.S. DOE by the UC LLNL and LANL under Contract No. W-7405-Eng-48. The quasi-isentropic compression experiments were conducted at the University of Rochester Laboratory for Laser Energetics under the NLUF grants program. Additional support was provided by LDRD program Project No. 06-SI-004 at LLNL and by the DOE under Grants No. DEFG0398DP00212 and No. DEFG0300SF2202, by the U.K. EPSRC under Grant No. GR/R25699/01.

\section{References}

[1] Tristan Guillot. The interiors of giant planets: Models and outstanding questions. Annual Review of Earth and Planetary Sciences, 33(1):493-530, 2005.

[2] S. J. Clark, G. J. Ackland, and J. Crain. Theoretical stability limit of diamond at ultrahigh pressure. Physical Review B, 52(21):15035-15038, 1995.

[3] T. Guillot. Interiors of giant planets inside end outside the solar system. Science, 286(5437):7277, 1999. 
[4] B. A. Remington, R. M. Cavallo, M. J. Edwards, D. D. M. Ho, B. F. Lasinski, K. T. Lorenz, H. E. Lorenzana, J. M. McNaney, S. M. Pollaine, and R. F. Smith. Accessing high pressure states relevant to core conditions in the giant planets. Astrophysics and Space Science, 298(12):235-240, 2005.

[5] D.H. Kalantar, E. Bringa, M. Caturla, J. Colvin, K. T. Lorenz, M. Kumar, J. Stolken, A. M. Allen, K. Rosolankova, J. S. Wark, M. A. Meyers, M. Schneider, and T. R. Boehly. Multiple film plane diagnostic for shocked lattice measurements (invited). Review of Scientific Instruments, 74:1929-1934, 2003.

[6] D. H. Kalantar, J. Belak, E. Bringa, K. Budil, M. Caturla, J. Colvin, M. Kumar, K. T. Lorenz, R. E. Rudd, J. Stolken, A. M. Allen, K. Rosolankova, J. S. Wark, M. A. Meyers, and M. Schneider. High-pressure, high-strain-rate lattice response of shocked materials. Physics of Plasmas, 10(5):1569-1576, 2003.

[7] A. Loveridge-Smith, A. Allen, J. Belak, T. Boehly, A. Hauer, B. Holian, D. Kalantar, G. Kyrala, R. W. Lee, P. Lomdahl, M. A. Meyers, D. Paisley, S. Pollaine, B. Remington, D. C. Swift, S. Weber, and J. S. Wark. Anomalous elastic response of silicon to uniaxial shock compression on nanosecond time scales. Phys. Rev. Lett., 86(11):2349-2352, 2001.

[8] S. K. Saxena and L. S. Dubrovinsky. Iron phases at high pressures and temperatures; phase transition and melting. American Mineralogist, 85(2):372-375, 2000.

[9] J. C. Boettger and D. C. Wallace. Metastability and dynamics of the shock-induced phase transition in iron. Physical Review B, 55(5):2840-2849, 1997.

[10] C. S. Yoo, N. C. Holmes, M. Ross, D. J. Webb, and C. Pike. Shock temperatures and melting of iron at earth core conditions. Phys. Rev. Lett., 70(25):3931-3934, 1993.

[11] Marc Andre Meyers. Dynamic behavior of materials. New York Wiley, 1994.

[12] James R. Asay. The use of shock-structure methods for evaluating high-pressure material properties. International Journal of Impact Engineering, 20(1-5):27, 1997.

[13] C. A. Hall. Isentropic compression experiments on the sandia z accelerator. Physics of Plasmas, $7(5): 2069-2075,2000$.

[14] J. F. Barnes, P. J. Blewett, R. G. Mcqueen, K. a. Meyer, and D. Venable. Taylor instability in solids. Journal of Applied Physics, 45(2):727-732, 1974.

[15] J. Edwards, K. T. Lorenz, B. A. Remington, S. Pollaine, J. Colvin, D. Braun, B. F. Lasinski, D. Reisman, J. M. McNaney, J. A. Greenough, R. Wallace, H. Louis, and D. Kalantar. Laserdriven plasma loader for shockless compression and acceleration of samples in the solid state. Phys. Rev. Lett., 92(7):-, 2004.

[16] K. T. Lorenz, M. J. Edwards, S. G. Glendinning, A. F. Jankowski, J. McNaney, S. M. Pollaine, and B. A. Remington. Accessing ultrahigh-pressure, quasi-isentropic states of matter. Physics of Plasmas, 12(5):-, 2005. 
[17] D. H. Kalantar, E. A. Chandler, J. D. Colvin, R. Lee, B. A. Remington, S. V. Weber, L. G. Wiley, A. Hauer, J. S. Wark, A. Loveridge, B. H. Failor, M. A. Meyers, and G. Ravichandran. Transient x-ray diffraction used to diagnose shock compressed si crystals on the nova laser. Review of Scientific Instruments, 70:629-632, 1999.

[18] Y. M. Gupta, K. A. Zimmerman, P. A. Rigg, E. B. Zaretsky, D. M. Savage, and P. M. Bellamy. Experimental developments to obtain real-time x-ray diffraction measurements in plate impact experiments. Review of Scientific Instruments, 70(10):4008-4014, 1999.

[19] D. Bancroft, E. L. Peterson, and S. Minshall. Polymorphism of iron at high pressure. Journal of Applied Physics, 27(3):291-298, 1956.

[20] J.M. Walsh. Bulletin of the American Physical Society, 29:28, 1954.

[21] P. W. Bridgman. Collected experimental papers. Harvard University Press, Cambridge,, 1964. [by] P.W. Bridgman. illus. $25 \mathrm{~cm}$. Some papers in German. "In most cases the original reprints have been reproduced photographically, although in a few instances it proved necessary to reset a paper in type.".

[22] J.C. Jamieson and A. W. Lawson. X-ray diffraction studies in the 100 kilobar pressure range. Journal of Applied Physics, 33(3):776-780, 1962.

[23] D. H. Kalantar, J. F. Belak, G. W. Collins, J. D. Colvin, H. M. Davies, J. H. Eggert, T. C. Germann, J. Hawreliak, B. L. Holian, K. Kadau, P. S. Lomdahl, H. E. Lorenzana, M. A. Meyers, K. Rosolankova, M. S. Schneider, J. Sheppard, J. S. Stolken, and J. S. Wark. Direct observation of the alpha-epsilon transition in shock-compressed iron via nanosecond $\mathrm{x}$-ray diffraction. Phys. Rev. Lett., 95(7):075502, 2005. 